\title{
Long-term persistence of MRSA in re-admitted patients
}

\author{
F. Mattner $\cdot$ F. Biertz $\cdot$ S. Ziesing $\cdot$ P. Gastmeier $\cdot$ \\ I. F. Chaberny
}

Received: 14 February 2010/ Accepted: 9 June 2010/Published online: 3 July 2010

(C) The Author(s) 2010. This article is published with open access at Springerlink.com

\begin{abstract}
Background A better knowledge of methicillin-resistant Staphylococcus aureus (MRSA) persistence in hospitalised patients may impact on specific prevention strategies. We have investigated the persistence of MRSA-carriage in patients admitted and re-admitted to a university hospital. Patients and methods Between January 2002 and October 2005 all MRSA-positive patients admitted to the university hospital of Hannover Medical School were assessed at first admission and all subsequent re-admissions. Patients readmitted at least once were analysed for the persistence or loss of MRSA. The association of possible factors influencing the persistence of MRSA colonisation or infection (age group, gender, decolonisation therapy during first hospital stay due to MRSA positivity and colonisation of different anatomical sites) was analysed using univariate, multivariate and time-dependent analyses.

Results A total of 1,032 patients who had tested positive at least once for MRSA were admitted to our hospital during the study period, accounting for 2,038 admissions. Of these patients, $403(39.1 \%)$ were admitted more than
\end{abstract}

Present Address:

F. Mattner $(\bowtie)$

Zentralbereich Hygiene, Kliniken der Stadt Köln,

Universitätsklinikum Witten-Herdecke, Campus Köln-Merheim,

Ostmerheimer Straße 200, 51107 Cologne, Germany

e-mail: mattnerf@kliniken-koeln.de

F. Biertz

Institute for Biometrics, Hannover Medical School,

Carl-Neuberg-Strasse 1, 30625 Hannover, Germany

F. Mattner - S. Ziesing - P. Gastmeier - I. F. Chaberny Institute for Medical Microbiology and Hospital Epidemiology, Hannover Medical School, Carl-Neuberg-Strasse 1, 30625 Hannover, Germany once (from two times to 21 times), and 238 (59.1\%) of the re-admitted patients remained MRSA positive during all subsequent admissions. Fifty-five (13.6\%) patients tested MRSA negative at their last admission, and 61 (15.1\%) tested MRSA negative at at least two consecutive admissions. In 27 (6.7\%) patients, the MRSA status differed more than once between subsequent admissions. Overall, the half-life time (HLT) of MRSA persistence was 549 days, with the duration of persistence dependent on the colonisation of different anatomical sites (HLT only wounds 117 days; HLT mouth, throat, bronchial secretions 627 days; HLT nose, wounds and other body sites 801 days; $p<0.01$ ) and was prolonged if more than one body site was MRSA-positive (HR 2.18, 95\% confidence interval 1.52-3.15).

Conclusion A detailed knowledge of the dynamics of the loss of MRSA infection could result in a reduction of the incidence of MRSA in the future. Multiple anatomical site carriage of MRSA appeared to predict a prolonged persistence in our cohort of patients re-admitted to a university hospital.

Keywords MRSA $\cdot$ Persistence $\cdot$ Re-admitted patients

\section{Introduction}

The spread of methicillin-resistant Staphylococcus aureus (MRSA) in North America and Europe has increased morbidity and mortality among patients admitted to hospitals as well as the costs of hospital stays over the past 30 years [1-10]. As MRSA is known to be transmitted mostly by health-care contacts $[7,11,12]$, it is important to determine whether initially MRSA-positive patients can lose their MRSA colonisation and to which extent and 
which factors influence the loss of MRSA. Existing data addressing the persistence of MRSA in patients hospitalised at least once describe half-life persistence times between 225 and 360 days, depending on the patient cohort investigated and the different decolonisation treatment applied [13-16]. Knowledge of the duration of MRSA persistence should impact on recommendations to flag patients who have once tested positive for MRSA in terms of testing them at re-admissions, but to date no recommendation exists regarding how long a patient should be flagged as an infection-control risk [17, 18]. In the study reported here, we investigated all patients admitted to a university hospital who had tested positive for MRSA at least once during slightly less than a 4-year period. Our aim was to determine MRSA persistence of the first and subsequent hospital stays and the time dependency of the loss of MRSA. We also analysed whether a topical decolonisation therapy during the first admission favoured or hindered the loss of MRSA and to what extent the MRSA loss was dependent on colonised anatomical sites.

\section{Methods}

Study design and setting

A cohort study was performed from January 2002 to October 2005 that included all detected MRSA-positive patients (either by clinical specimen or by screening culture) admitted to the university hospital of the Hannover Medical School (MHH) during the study period. The MHH hospital has 1,400 beds, with nearly 45,000 patient admissions per year, accounting for 400,000 patient-days.

Prospective surveillance of MRSA-positive patients

Diagnosis of a first positive microbiological result for MSRA at any anatomical site resulted in the patient being entered in our surveillance system, and all patient data being entered into a database that serves as an alert system in the case of subsequent re-admissions [19]. For every patient with a MRSA-positive test result, additional swabs of the nose, throat and wounds if present were recommended to estimate the extent of MRSA colonisation. An infection control nurse visited each patient, recommending a decolonisation therapy [mupirocin nasal ointment 3 times per day to both nares for 5 days, daily body and throat washings for 5 days with disinfectants containing octenidine (octenisept) or chlorhexidine (skinsept mucosa)]. The physician in charge decided if a decolonisation therapy should be initiated, and if initiated, the topical decolonisation therapy was recorded in the database. A systemic decolonisation therapy was not recommended. MRSA targeting antibiotic therapy was only initiated if infections were present.

Definition of a negative MRSA status

We defined an initially MRSA-positive patient as "MRSA negative" if three specimens taken at the anatomical sites that had originally tested MRSA positive, tested MRSA "negative" on at least 3 subsequent days. In addition, nares were tested for MRSA. Negative results were only accepted if MRSA susceptible antibiotics were stopped at least 2 days before the first swab was taken.

\section{Microbiological diagnostic of MRSA}

All clinical specimens were cultured according to routine microbiological diagnostic procedures on 5\% sheep blood agar (BBL Microbiology Systems, Becton-Dickinson, Heidelberg, Germany) and mannitol salt agar (own production). The antibiotic susceptibility of $S$. aureus isolates was measured by broth micro dilution with the MICRO$\mathrm{NAUT}^{\mathrm{TM}}$ system (Merlin Diagnostika GmbH, Hensel, Germany).

From July 2004 to October 2005, only screening swabs were cultured directly onto a selective culture medium (MRSA ID-Agar ${ }^{\text {TM}}$; bioMerieux, Marcy l'Etoile, France). Susceptiblity interpretations were made according to breakpoints in accordance with the Deutsche Ingenieur Norm (DIN) 58940-4.

Isolates were confirmed using an agglutination test for penicillin-binding protein 2a (PBP'2 Latex Agglutiniation kit; bioMérieux).

MRSA status of re-admitted patients

All MRSA-positive discharged patients had to be re-examined upon re-admission at all of the initially MRSA-positive anatomical sites and the nose and throat. If these were missed (42 cases, 10.4\%), the patient was regarded as "MRSA-positive". In contrast to Robiczek et al. [14], we preferred to overestimate rather than underestimate the MRSA persistence in order to maximise the safety of possible recommendations regarding how long MRSA-positive patients should be flagged.

Observation period of initially MRSA-positive patients

For every MRSA-positive patient that had been entered into the database, all subsequent admissions during the study period were included in our study. The microbiological database was retrospectively checked for MRSApositive as well as for any MRSA-negative specimens for all patients. The overall observation period of each patient 
was determined by subtracting the admission date of the first admission during which the first positive MRSA result was detected from the date of discharge of the last hospital admission. Patients who had once tested "MRSA negative" were not screened at re-admission for reasons of their initial MRSA positivity, although they could be screened as a result of belonging to a high risk group. Patients who were screened could be detected as MRSA positive once again based on laboratory testing of clinical specimens.

Determination of the anatomical MRSA colonisation site of the patient

We grouped all patients into the following distinct colonisation groups to enable an analysis of MRSA-site dependent persistence, with each different anatomical site that tested MRSA positive considered independent of the time point when the detection was made: (1) only nares; (2) only wounds; (3) nares and other anatomical sites but no wounds; (4) throat, mouth, tracheal secretion, bronchial secretion or bronchoalveolar lavage (BAL) without nares; (5) nares and other anatomical sites including wounds; (6) other anatomical sites, including urinary tract and anogenital region without nares and without wounds; (7) wounds and other anatomical sites without nares; (8) any other anatomical sites and combinations which are not already described above.

Determination of the MRSA status of a patient at the end of a hospital stay

The MRSA status of a patient was determined as "positive" in any case in which no three negative samples of an initially MRSA-positive anatomical site or nares could be obtained on at least three subsequent days-under the condition that no MRSA-susceptible antibiotics had been given.

Definition of the patient cohorts for the MRSA persistence analysis (univariate analysis, Kaplan-Meier survival analysis, Cox proportional hazards models)

All patients who had tested MRSA positive at least once and had been re-admitted at least once during the study period were included in our study. The first day of the colonisation time was determined as either the admission date (if the patient had positive MRSA results from another laboratory) or the date of the first MRSA-positive specimen. The last day was defined as the day on which the first of three MRSA-negative specimens was diagnosed according to our definition of a MRSA-negative status in the case of patients losing the MRSA infection and the date of the last hospital discharge during the study period for patients remaining MRSA-positive.

For the Kaplan-Meier analysis and Cox proportional hazards models, all patients who changed MRSA status between admissions at least twice (e.g. positive-negativepositive pattern during subsequent admissions) were excluded from the analysis. We elected to perform a timedependent analysis even though the data were interval censored (the exact day of MRSA loss between two hospital stays can not be determined). The resulting overestimation of MRSA persistence is adequate for defining safe intervals for how long MRSA-positive patients should be flagged after discharge.

The Cox proportional hazard models analysis for the time until loss was performed using the variables "age $\leq 65$ years" and "topical decolonisation therapy" as dichotome variables and "anatomical sites of colonisation" as parametric variables. All variables were included in the final model.

\section{Statistical analysis}

Fisher's exact test or chi-square tests, log rank sum tests, Kaplan-Meier survival analysis and Cox proportional hazards models analysis were performed as appropriate using SPSS software ver. 15.0 (SPSS, Chicago, IL). $P$ values $<0.05$ were regarded as significant.

\section{Results}

A total of 1,032 patients who had tested positive at least once for MRSA were admitted to our hospital during the study period, accounting for 2,038 admissions. Of these, 403 patients were admitted more than once (range: 2-21 times), accounting for 1,409 admissions. Microbial specimens adequate for MRSA detection were available for $84 \%$ of all admissions $(2,038)$; the remaining $16 \%$ were either re-admissions or first admissions (20 patients) for which the MRSA have been tested "positive" by external laboratories. In the first case, the original MRSA status for the patient remained unchanged (i.e. was the same as their former hospital admission). Per admission, a median of nine (4: $25 \%$ percentile, $21: 75 \%$ percentile) microbial cultures of a median of three (2: $25 \%$ percentile; $5: 75 \%$ percentile) different body sites were performed. Table 1 shows how many patients remained MRSA positive, how many lost their MRSA-positive status and how many switched between a MRSA-positive and MRSA-negative status between different admissions as well as the frequency of MRSA-"positive" tested anatomical sites, the initiation of a decolonisation therapy at the first MRSApositive admission and colonisation of either one or more 
Table 1 Study cohort including all patients who had tested MRSA positive at least once and had been re-admitted at least once (Patients' characteristics/anatomical colonisation sites/topical decolonisation)
SD Standard deviation, MRSA methicillin-resistant Staphylococcus aureus, BAL bronchoalveolar lavage

${ }^{a}$ Data from patients available for anatomical site determination, comprising results of screening cultures for nares, throats and wounds and cultures of clinical specimens

${ }^{\mathrm{b}}$ Only patients with available information for decolonisation were included $(n=394)$

\begin{tabular}{|c|c|}
\hline Characteristics & $\begin{array}{l}\text { Number (\%; unless otherwise } \\
\text { stated) }\end{array}$ \\
\hline Number of patients & 403 \\
\hline Male & 257 \\
\hline Age $>65$ years & 121 \\
\hline Age (years): (mean $\pm \mathrm{SD}$, median, range; 25-, 75-percentiles) & $\begin{array}{l}52.9 \pm 19.7,57.0,0-92 ; 41.0 \\
\quad 68.0\end{array}$ \\
\hline $\begin{array}{l}\text { Duration of observation period (days): (mean } \pm \mathrm{SD} \text {, median, } \\
\text { range; and 25-, 75- percentiles) }\end{array}$ & $\begin{array}{l}301.2 \\
435\end{array}$ \\
\hline \multicolumn{2}{|l|}{$\begin{array}{l}\text { MRSA status of patients at the time of discharge of their last observed } \\
\text { admission during the study period }\end{array}$} \\
\hline At all subsequent discharges MRSA-positive & $238(59.1 \%)$ \\
\hline Only at the last observed re-admission MRSA-negative & $55(13.6 \%)$ \\
\hline At the last observed at least two re-admissions MRSA-negative & $61(15.1 \%)$ \\
\hline $\begin{array}{l}\text { At one admission MRSA-negative followed by re-admission(s) } \\
\text { with no confirmation of MRSA negativity }\end{array}$ & $22(5.4 \%)$ \\
\hline $\begin{array}{l}\text { At subsequent admissions switching MRSA status (positive- } \\
\text { negative-positive, positive-negative-positive-negative-positive) }\end{array}$ & $27(6.7 \%)$ \\
\hline \multicolumn{2}{|l|}{ Patients with MRSA colonisations at anatomical site groups ${ }^{\mathrm{a}}$} \\
\hline Only nares & $48(11.95 \%)$ \\
\hline Nares and other anatomical sites but no wounds & $114(28.3 \%)$ \\
\hline Nares and other anatomical sites including wounds & $90(22.3 \%)$ \\
\hline $\begin{array}{l}\text { Throat, mouth, tracheal secretion, bronchial secretion or BAL } \\
\text { without nares }\end{array}$ & $38(9.4 \%)$ \\
\hline Only wounds & $21(5.2 \%)$ \\
\hline Wounds and other anatomical sites without nares & $38(9.4 \%)$ \\
\hline $\begin{array}{l}\text { Other anatomical sites including urinary tract and ano-genital } \\
\text { region without nares and without wounds }\end{array}$ & $34(8.4 \%)$ \\
\hline $\begin{array}{l}\text { Any other anatomical sites which are not already described } \\
\text { above }\end{array}$ & $19(6.7 \%)$ \\
\hline Unknown colonisation site & $1(0.2 \%)$ \\
\hline Single anatomical colonisation site & $84(20.9 \%)$ \\
\hline Multiple anatomical colonisation site & $318(79.1 \%)$ \\
\hline $\begin{array}{l}\text { Topical decolonisation therapy initiated during the first MRSA-positive } \\
\text { admission }\end{array}$ & $153(38.8 \%)$ \\
\hline
\end{tabular}

anatomical sites. Of 138 patients, 61 who had become MRSA negative were screened again at least on of their readmissions. Fifty-five MRSA-negative patients were not re-admitted, and screening cultures during re-admission were missing for 22 patients.

The over-all half-life time (HLT) of MRSA persistence was 566 days for a patient who had been re-admitted at least once. To detect a potential bias by different readmission frequencies between the MRSA-persistence and MRSA-loss group, we tested whether these groups differed by re-admission frequency. However, no significant difference was found (data not shown).

Patients with a MRSA colonisation at anatomical sites "nares and others excluding wounds" had about a threefold increased duration of MRSA persistence (HLT 630 days) compared to patients colonised at the anatomical site "only nares" (HLT 197 days) or "only wounds" (HLT 123 days) (Fig. 1). Patients colonised at "throat, mouth, tracheal secretion, bronchial secretion or BAL without nares" (HLT 576 days) and those colonised at "other anatomical sites including urinary tract and ano-genital region without nares or wounds" (HLT 387 days) showed an increased MRSA persistence.

Both the univariate and Kaplan-Meier analyses revealed that gender was not a significant risk factor for prolonged MRSA persistence (HLT 566 days for males, 420 days for females; $p=0.90)$. Patients in the age groups $\leq 65$ and $>65$ years, respectively, did not differ in MRSA persistence (HLT 554 vs. 549 days, respectively; $p=0.18)$.

Patients colonised at only one anatomical site had a significantly shorter MRSA persistence than those 


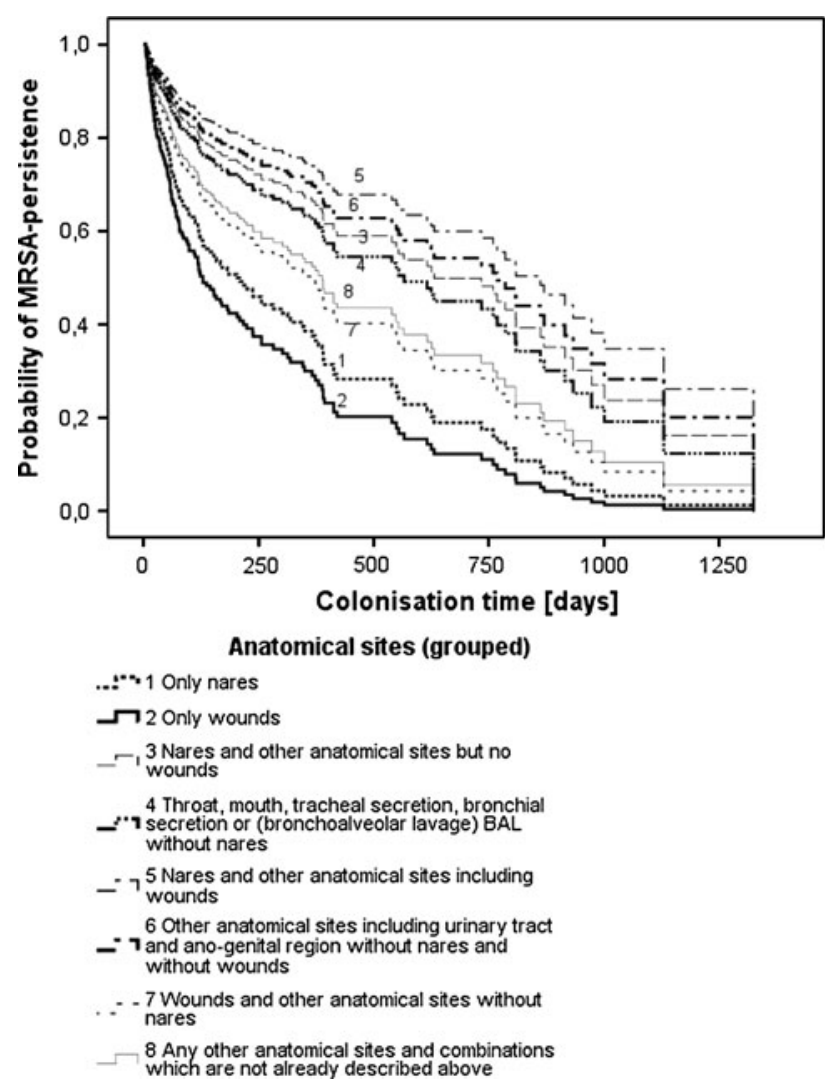

Fig. 1 Methicillin-resistant Staphylococcus aureus (MRSA) persistence in patients re-admitted at least once according to MRSA colonisations at different anatomical sites. (For this analysis, 27 (6.7\%) patients with at least two changes in MRSA status between hospital stays were excluded). $p$ ( $\chi^{2}$-test $)<0.01$

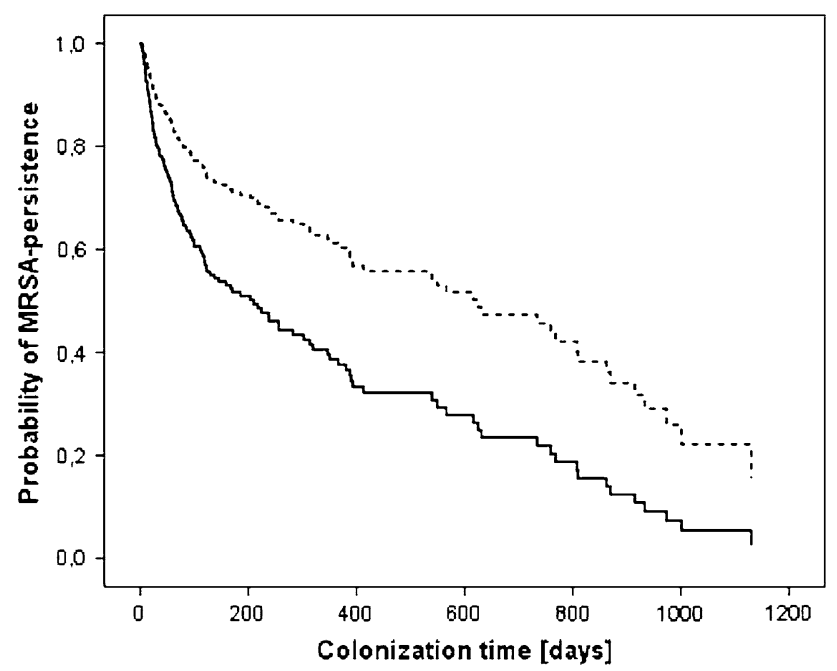

Fig. 2 MRSA persistence according to colonisation at single or at multiple anatomical sites [28 (6.9\%) patients with unknown anatomical site colonisation were excluded]. Hazard ratio 2.18, 95\% confidence interval $1.52-3.15, p<0.01$. Thick line Colonisation at a single anatomical site, dotted line colonisation at multiple anatomical sites colonised at multiple anatomical sites [see Fig. 2; hazard ratio (HR) 2.18, 95\% confidence interval (CI) 1.52-3.15; $p\left(\chi^{2}\right.$-test $\left.)<0.01\right]$.

The effect of a topical decolonisation therapy during the first admission was tested after the exclusion of all patients with alternating MRSA status. The univariate analysis revealed a significant effect of topical decolonisation therapy for the first admission [odds ratio (OR) 4.01, 95\% CI 1.69-9.48, $p<0.01]$. In contrast, the time-dependent analysis (Kaplan-Meier and Cox proportional hazards models) revealed that the effect of decolonisation therapy during the first MRSA-positive admission was not significant (HLT 413 vs. 734 days; $p=0.20$ ).

Multivariate data analysis (Cox proportional hazards models) including the variables "anatomical site of colonisation", "age at first stay ( $\leq 65 />65$ years)" and "decolonisation during the first stay" revealed a significant association of the MRSA persistence to colonisation of different anatomical sites (Table 2).

\section{Discussion}

The admittance of MRSA-colonised patients to hospitals represent a major challenge to healthcare providers charged with ensuring the safety of patient care in hospitals [20]. As MRSA is easily transmitted in hospitals, expensive isolation precautions are recommended to prevent further nosocomial spread [18]. The aim of our study was to determine the probability that patients once hospitalised for MRSA and then re-admitted would lose the MRSA infection again over the long term. To this end, we followed our MRSA cohort over a number of years and observed MRSA colonisation or loss from re-admission to re-admission.

Almost $40 \%$ of our MRSA-positive patients were re-admitted to our hospital, underlining that only an automated alert system enables the reliable recognition of already known MRSA-positive patients and, thus, the immediate initiation of MRSA-transmission prevention measures.

Our investigation revealed that one-fourth of our cohort lost the MRSA infection after about 4 months; after about 18 months, half of the cohort was MRSA-free. Due to their decision to underestimate MRSA persistence rather than overestimate it (as we did), the durations recently reported by Robiczek et al. were shorter than those reported here [14]. In contrast to a number of other studies, our study cohort comprised only patients treated in and re-admitted to a university hospital, which implies that the patients were possibly sicker than those in other studies (Table 3). In addition, we did not perform any follow-up during ambulatory care. Again, the resulting selection and detection bias of our study cohort also result in an 
Table 2 Multivariate analysis of MRSA loss according to age group, decolonisation therapy at first hospital stay, and MRSA colonisation at different anatomical sites

\begin{tabular}{|c|c|c|c|}
\hline \multirow[t]{2}{*}{ Variables } & \multicolumn{2}{|c|}{ Patients re-admitted at least once } & \multirow[t]{2}{*}{$p$ value } \\
\hline & $\begin{array}{l}\text { HR of } \\
\text { MRSA loss }\end{array}$ & $\begin{array}{l}95 \% \mathrm{CI} \\
\text { for } \mathrm{HR}\end{array}$ & \\
\hline $\begin{array}{l}\text { Age at first hospital stay (years) } \\
(>65 / \leq 65 \text { years })\end{array}$ & 0.87 & $0.57-1.33$ & 0.53 \\
\hline $\begin{array}{l}\text { Topical decolonisation therapy initiated during the } \\
\text { first hospital stay: Yes/no }\end{array}$ & 0.75 & $0.51-1.11$ & 0.15 \\
\hline Anatomical site (total) & - & - & $<0.01$ \\
\hline Only wounds/only nares & 1.63 & $0.69-3.86$ & 0.26 \\
\hline Nares and other anatomical sites but no wounds/only nares & 0.49 & $0.28-0.88$ & 0.02 \\
\hline $\begin{array}{l}\text { Throat, mouth, tracheal secretion, bronchial secretion or BAL } \\
\text { without nares/only nares }\end{array}$ & 0.65 & $0.33-1.28$ & 0.22 \\
\hline Nares and other anatomical sites including wounds/only nares & 0.34 & $0.19-0.63$ & $<0.01$ \\
\hline $\begin{array}{l}\text { Other anatomical sites including urinary tract and ano-genital } \\
\text { region without nares and without wounds/only nares }\end{array}$ & 0.47 & $0.21-1.06$ & 0.07 \\
\hline Wounds and other anatomical sites without nares/only nares & 0.83 & $0.41-1.67$ & 0.59 \\
\hline Any other anatomical sites or combinations/only nares & 0.76 & $0.35-1.66$ & 0.49 \\
\hline Unknown/only nares & 3.44 & $0.45-26.39$ & 0.24 \\
\hline
\end{tabular}

$H R$ Hazard ratio, $C I$ confidence interval

overestimation of MRSA persistence. The long period of MRSA persistence among out study cohort demonstrates that formerly hospitalised MRSA-positive patients may also impact on patient care facilities for out-patients. Therefore, a diagnostic, therapeutic and transmission preventive management programme for these patients is warranted in the context of ambulatory care. The first step is the implementation of MRSA networks [21]. On the other hand, long-term observation periods for MRSA holds some interest because a consequent prevention of MRSA transmission in hospitals would result in a natural reduction of MRSA-positive patients over the years if no additional cases were to occur. Our data also suggest that MRSApositive patients should not only be entered in an alert system but also redrawn when the MRSA infection is lost. This implies that an MRSA alert database should be updated on a regular basis. Of course, confidence in the method of just how to assess that the MRSA is "lost" is always limited. However, in our study, we required three negative microbiological results for the initial MRSApositive anatomical sites, nares, throat and any wounds in the absence of antibiotic treatment effective against MRSA and on at least three different days. All patients from whom we obtained less than three negative specimens of the respective colonisation sites were considered to be MRSA "positive" even if the materials obtained were MRSAnegative. Thus, we are rather sure that the cohort that became MRSA negative was at most underestimated and that the cohort with MRSA persistence might have been overestimated. In addition, we observed that we had only a rather small patient cohort of about $6 \%$ who changed their MRSA status between different admissions at least twice, which is consistent with data published by Vriens et al. [16]. We had insufficient data to determine whether our MRSA-negative patients would be recolonised after a longer period during ambulatory care. This important question should be addressed in future studies using a study design with an active controlled screening protocol.

The effects of a decolonisation treatment that have been reported to date are conflicting [13, 22-25]. We found a significant effect of one topical decolonisation therapy during the patient's first MRSA-positive hospital stay even though we could not control the adherence to the decolonisation protocol (data not shown in detail). However, we were unable to confirm a clearly significant effect of one initial topical decolonisation therapy over the long term (considering only patients being re-admitted at least once) as described by Marschall et al. [13]. As we categorised all patients who did not fulfil our "MRSA-negative" requirements as "MRSA-positive" to exclude a possible overestimation of any effect, we can not exclude the possibility that we underestimated the possible effect of a decolonisation therapy in our cohort. Thus, based on our results, the effectiveness of topical decolonisation therapy remains an unresolved question, even though recent publications report a significant advantage if the decolonisation treatments are rigorously controlled and a systemic therapy is partially added [22].

As the risk of infection seems to depend on the initially colonised anatomical site, it has also been suspected that 


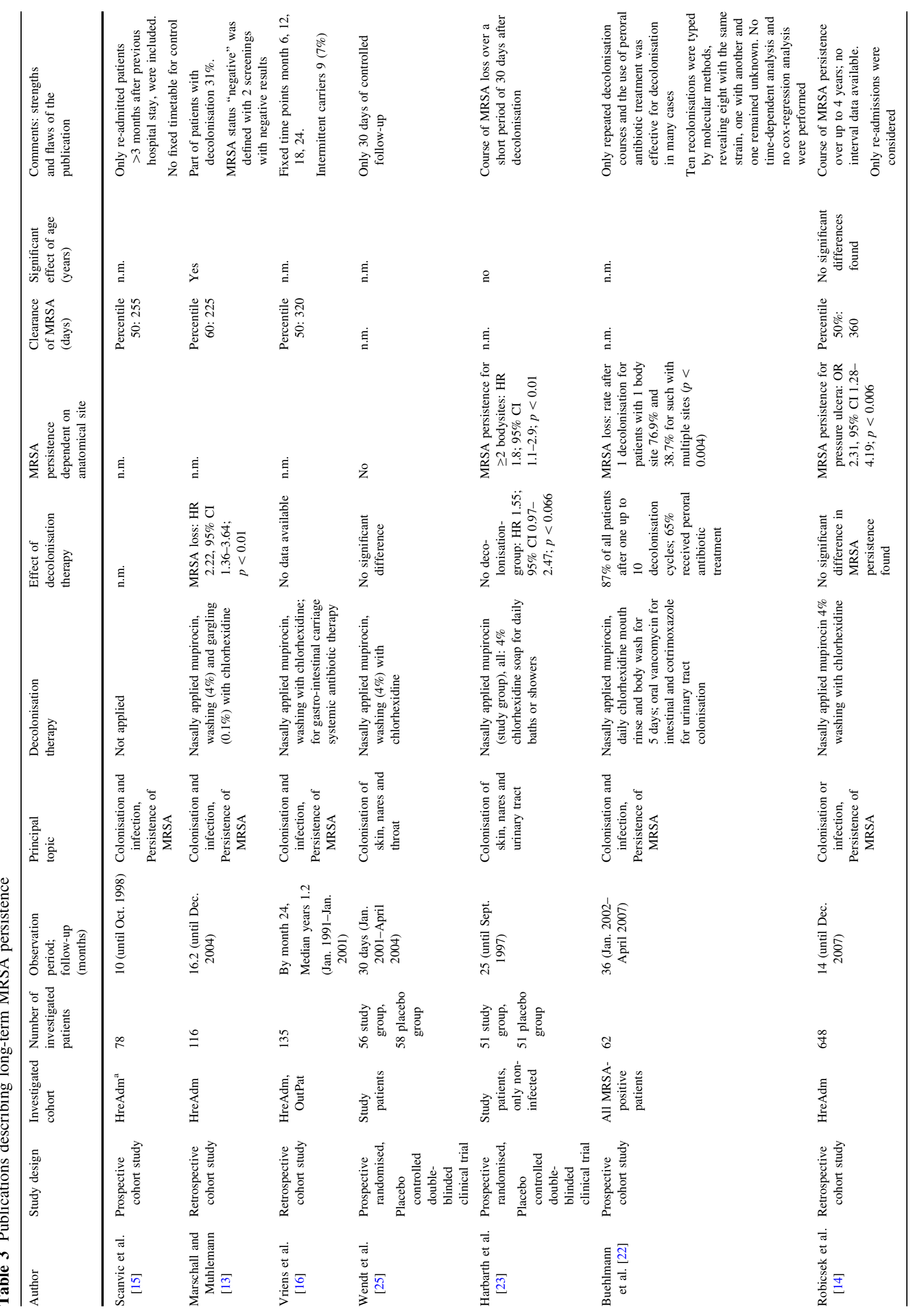


the loss of MRSA depends on the kind of anatomical sites initially colonised [20]. We therefore calculated the risks according to several anatomical site categories and found that the loss or persistence differed significantly. In addition, MRSA persistence was prolonged if more than one anatomical site tested positive, which is consistent to the findings reported by Buehlmann et al. [22]. Our data suggest starting decolonisation therapy as early as possible after MRSA has been detected and to focus on patients who are colonised at only one or few different body sites.

Unfortunately our data are limited by a certain incompleteness of microbiological follow-up due to the observational nature of our study with no defined interval screening protocol for MRSA. Hence, we cannot exclude the possibility that patients colonised at various anatomical sites were only detected due to an increased severity of illness or that patients found colonised at only few anatomical sites were biased by missing screening cultures of additional anatomical body sites.

Only a few published studies have addressed the question of MRSA persistence over the long term, and all of these studies differ in methodolgy and study design (see Table 3) [13-15, 26].

Most studies carried out to date, including our study, are limited by the fact that there is no information on the MRSA status of patients between different admissions. This leads to the problem of interval censoring, which we could not solve. This problem requires further studies that include data from ambulatory patient care.

\section{Conclusions}

A high percentage-about $40 \%$-of all MRSA-positive patients was re-admitted at least once. At least $15 \%$ of all patients lost MRSA colonisation over time. The HLT of MRSA in our selected patient cohort appears to be prolonged, with a HLT of about 560 days. Multiple-site carriage seems to determine a prolonged carriage of MRSA. The shortest MRSA persistence times were found in patients who were only colonised in wounds.

Acknowledgments We acknowledge the following members of the infection control team for their excellent assistance: D. RotermundRauchenberger, U. Schöner-Frühling, U. Jacobi and J. Bruderek. We want to thank M. to Baben-Yang for the management of the MRSA alert database.

\section{Conflict of interest statement None.}

Open Access This article is distributed under the terms of the Creative Commons Attribution Noncommercial License which permits any noncommercial use, distribution, and reproduction in any medium, provided the original author(s) and source are credited. 


\section{References}

1. Tiemersma EW, Bronzwaer SL, Lyytikainen O, et al. Methicillinresistant Staphylococcus aureus in Europe, 1999-2002. Emerg Infect Dis. 2004;10:1627-34.

2. Tietz A, Frei R, Widmer AF. Transatlantic spread of the USA300 clone of MRSA. N Engl J Med. 2005;353:532-3.

3. Witte W, Kresken M, Braulke C, Cuny C. Increasing incidence and widespread dissemination of methicillin-resistant Staphylococcus aureus (MRSA) in hospitals in central Europe, with special reference to German hospitals. Clin Microbiol Infect. 1997;3:414-22.

4. Chaberny IF, Ziesing S, Mattner F, et al. The burden of MRSA in four German university hospitals. Int $\mathrm{J}$ Hyg Environ Health. 2005;208:447-53.

5. Cosgrove SE, Sakoulas G, Perencevich EN, Schwaber MJ, Karchmer AW, Carmeli Y. Comparison of mortality associated with methicillin-resistant and methicillin-susceptible Staphylococcus aureus bacteremia: a meta-analysis. Clin Infect Dis. 2003;36:53-9.

6. Wernitz MH, Keck S, Swidsinski S, Schulz S, Veit SK. Cost analysis of a hospital-wide selective screening programme for methicillin-resistant Staphylococcus aureus (MRSA) carriers in the context of diagnosis related groups (DRG) payment. Clin Microbiol Infect. 2005;11:466-71.

7. Trautmann M, Pollitt A, Loh U, et al. Implementation of an intensified infection control program to reduce MRSA transmissions in a German tertiary care hospital. Am J Infect Control. 2007;35:643-9.

8. Davis KA, Stewart JJ, Crouch HK, Florez CE, Hospenthal DR. Methicillin-resistant Staphylococcus aureus (MRSA) nares colonization at hospital admission and its effect on subsequent MRSA infection. Clin Infect Dis. 2004;39:776-82.

9. Whitby M, McLaws ML, Berry G. Risk of death from methicillin-resistant Staphylococcus aureus bacteraemia: a meta-analysis. Med J Aust. 2001;175:264-7.

10. Cosgrove SE, Qi Y, Kaye KS, Harbarth S, Karchmer AW, Carmeli Y. The impact of methicillin resistance in Staphylococcus aureus bacteremia on patient outcomes: mortality, length of stay, and hospital charges. Infect Control Hosp Epidemiol. 2005;26: $166-74$.

11. Grundmann H, Hori S, Winter B, Tami A, Austin DJ. Risk factors for the transmission of methicillin-resistant Staphylococcus aureus in an adult intensive care unit: fitting a model to the data. $\mathrm{J}$ Infect Dis. 2002;185:481-8.

12. Thompson RL, Cabezudo I, Wenzel RP. Epidemiology of nosocomial infections caused by methicillin-resistant Staphylococcus aureus. Ann Intern Med. 1982;97:309-17.

13. Marschall J, Muhlemann K. Duration of methicillin-resistant Staphylococcus aureus carriage, according to risk factors for acquisition. Infect Control Hosp Epidemiol. 2006;27:1206-12.
14. Robicsek A, Beaumont JL, Peterson LR. Duration of colonization with methicillin-resistant Staphylococcus aureus. Clin Infect Dis. 2009;48:910-3.

15. Scanvic A, Denic L, Gaillon S, Giry P, Andremont A, Lucet JC. Duration of colonization by methicillin-resistant Staphylococcus aureus after hospital discharge and risk factors for prolonged carriage. Clin Infect Dis. 2001;32:1393-8.

16. Vriens MRBH, Gigengack-Baars AC, Mascini EM, van der Werken C, Verhoef J, Troelstra A. Methicillin-resistant Staphylococcus aureus carriage among patients after hospital discharge. Infect Control Hosp Epidemiol. 2005;26:629-33.

17. KRINKO (1999) Empfehlung zur Prävention und Kontrolle von Methicillin-resistenten Staphylococcus aureus-Stämmen (MRSA) in Krankenhäusern und anderen medizinischen Einrichtungen. Bundesgesundheitsblatt. 1999;(42):954-958.

18. Siegel JD, Rhinehart E, Jackson M, Chiarello L. Guideline for isolation precautions: preventing transmission of infectious agents in health care settings. Am J Infect Control. 2007;35:S65-164.

19. Chaberny IF, Schwab F, Ziesing S, Suerbaum S, Gastmeier P. Impact of routine surgical ward and intensive care unit admission surveillance cultures on hospital-wide nosocomial methicillinresistant Staphylococcus aureus infections in a university hospital: an interrupted time-series analysis. J Antimicrob Chemother. 2008;62:1422-9.

20. Huang SS, Platt R. Risk of methicillin-resistant Staphylococcus aureus infection after previous infection or colonization. Clin Infect Dis. 2003;36:281-5.

21. Daniels-Haardt I, Verhoeven F, Mellmann A, Hendrix MG, Gemert-Pijnen JE, Friedrich AW. EUREGIO-projekt MRSA-net Twente/Munsterland. Creation of a regional network to combat MRSA. Gesundheitswesen. 2006;68:674-8.

22. Buehlmann M, Frei R, Fenner L, Dangel M, Fluckiger U, Widmer AF. Highly effective regimen for decolonization of methicillin-resistant Staphylococcus aureus carriers. Infect Control Hosp Epidemiol. 2008;29:510-6.

23. Harbarth S, Liassine N, Dharan S, Herrault P, Auckenthaler R, Pittet D. Risk factors for persistent carriage of methicillin-resistant Staphylococcus aureus. Clin Infect Dis. 2000;31:1380-5.

24. Robicsek A, Beaumont JL, Thomson RB Jr, Govindarajan G, Peterson LR. Topical therapy for methicillin-resistant Staphylococcus aureus colonization: impact on infection risk. Infect Control Hosp Epidemiol. 2009;30:623-32.

25. Wendt C, Schinke S, Wurttemberger M, Oberdorfer K, BockHensley O, von Baum H. Value of whole-body washing with chlorhexidine for the eradication of methicillin-resistant Staphylococcus aureus: a randomized, placebo-controlled, double-blind clinical trial. Infect Control Hosp Epidemiol. 2007;28:1036-43.

26. Sanford MD, Widmer AF, Bale MJ, Jones RN, Wenzel RP. Efficient detection and long-term persistence of the carriage of methicillin-resistant Staphylococcus aureus. Clin Infect Dis. 1994;19:1123-8. 